\title{
Exponential stability of neutral stochastic functional differential equations driven by G-Brownian motion
}

\author{
Min Zhu ${ }^{\mathrm{a}, \mathrm{b}, *}$, Junping Li ${ }^{\mathrm{a}}$, Yongxiang Zhu ${ }^{\mathrm{b}}$ \\ a School of Mathematics and Statistics, Central South University, Changsha, Hunan, 410083, China. \\ ${ }^{b}$ College of Traffic Engineering, Hunan University of Technology, Zhuzhou, Hunan, 412007, China. \\ Communicated by $\mathrm{X}$. Liu
}

\begin{abstract}
In this work, we study a class of neutral stochastic functional differential equations driven by G-Brownian motion. We derive by variation-of-constants formula sufficient conditions for exponential stability and quasi sure exponential stability of the solutions. Finally, we provide an example to illustrate the effectiveness of the theoretical results. (c) 2017 All rights reserved.
\end{abstract}

Keywords: Neutral, variation-of-constants formula, exponential stability, G-Brownian motion. 2010 MSC: 60H20, 60H30.

\section{Introduction}

The theory of neutral functional differential equations has attracted many researchers' great attention for its potential applications in finance, population dynamic and control. For instance, Hale and Lunel [9] studied the fundamental theory of deterministic neutral functional differential equations. Liu [17] considered standard optimal control problems for a class of neutral functional differential equations. One of the basic works of the theory of neutral functional differential equations is to study the theory of stability of solutions. Moreover, there is an extensive literature on the stability of neutral stochastic functional differential equations (NSFDEs), such as [2, 3, 12, 15, 18-21, 28]. In particular, Mao [19] investigated the exponential stability in mean square for a NSFDEs. Mao also established several Razumikhin-type theorems on the exponential stability for stochastic functional differential equations in [20].

Meanwhile, the theory of nonlinear expectation has also drawn a great deal of attention in uncertainty problems, risk measures and the superhedging in finance. Especially, Peng [22] established the fundamental theory of the G-expectation and G-conditional expectation. Under the framework of G-expectation, Peng $[23,24]$ not only introduced the notion of G-Gaussian distribution and the G-Brownian motion but also set up the associated stochastic calculus of Itô type. On the basis of Peng's works, related topics in

\footnotetext{
*Corresponding author Zhu)

Email addresses: zhumin0107@csu.edu.cn (Min Zhu), jpli@mail.csu.edu.cn (Junping Li), zyx1998@sina.com (Yongxiang
}

doi:10.22436/jnsa.010.04.43 
the G-stochastic analysis are developed by many researchers, especially on the stability properties. For example, Zhang and Chen [27] investigated the sufficient conditions for quasi surely exponentially stability of the solutions for a kind of special G-stochastic differential equations (GSDEs). Fei and Fei [7] established the exponential stability of paths for a class of GSDEs. Hu et al. [11] studied p-moment stability of solutions to GSDEs by means of the Lyapunov function and the Itô formula. Ren et al. [26] established the p-moment exponential stability and quasi sure exponential stability of solutions to impulsive GSDEs by means of G-Lyapunov function method. Through more careful observation and analysis, readers can discover that G-Lyapunov function method is in general available to establish the stability of solutions to GSDEs.

Motivated by the above discussion, we will, in this present work, be interested in the stability problem for a class of neutral stochastic functional differential equations driven by G-Brownian motion (SFDEGs). In contrast to the existing results on GSDEs, by now there was no study of neutral SFDEGs. Only, Ren et al. [25] studied the existence and uniqueness of the solutions to SFDEGs with the coefficients satisfying both the linear growth condition and the classical Lipschitz condition, and investigated the exponential estimate of the solutions. However, they did not consider the stability of the solutions. The techniques used in [25] to prove the existence and uniqueness of the solutions are not suitable to the model whose coefficients do not satisfy the Lipschitz condition. Different from those result obtained in $[7,11,26,27]$, in this work we shall investigate the p-moment exponential stability and quasi sure exponential stability of solutions to a class of neutral SFDEGs. As well all know, in the case without delay, Lyapunov's method is in general available to study the stability of solutions to GSDEs, as seen in [7, 11, 26, 27]. However, in the case of differential equations with retarded arguments, even with constant delays, Lyapunov's method is not suitable as Krasovkii [13] pointed out for the ordinary differential equations, and Kushner [14] (among others) also did for stochastic differential equations (SDEs), since the history of the process must be taken into account [10]. In addition, the main method in the aforementioned references is to incorporate certain dissipativity to establish the stability of solutions to NSFDEs, as seen in $[15,19,28]$ and [3]. The dissipativity is assured by imposing information of the current time with certain decay conditions [5]. But this method cannot deal with the system in this work, since both the drift and diffusion coefficients involve only retarded elements and do not involve information on current time. All these issues will hinder the development of stochastic systems. Consequently, the stability of neutral SFDEGs should be developed.

\section{Preliminary}

Let $(\Omega, \mathcal{H}, \overline{\mathbb{E}})$ be a sublinear expectation space. Denote $\Omega=\mathrm{C}_{0}\left(\mathbb{R}^{+}\right)$by the space of all real-valued continuous paths $\left(\omega_{t}\right)_{t \in \mathbb{R}^{+}}$with $\omega_{0}=0$ equipped with the distance

$$
\rho\left(\omega^{1}, \omega^{2}\right)=\sum_{i=1}^{\infty} 2^{-i}\left[\left(\max _{t \in[0, i]}\left|\omega_{t}^{1}-\omega_{t}^{2}\right|\right) \wedge 1\right] .
$$

For every $\omega \in \Omega$, let $B(\cdot, \omega)=\omega(\cdot)$ be the corresponding canonical process. For each fixed $T \in[0, \infty)$, set

$$
\operatorname{Lip}\left(\Omega_{\mathrm{T}}\right):=\left\{\varphi\left(\mathrm{B}\left(\mathrm{t}_{1}\right), \mathrm{B}\left(\mathrm{t}_{2}\right), \ldots, \mathrm{B}\left(\mathrm{t}_{\mathrm{m}}\right)\right): \mathrm{m} \in \mathbb{N}, \mathrm{t}_{1}, \ldots, \mathrm{t}_{\mathrm{n}} \in[0, \mathrm{~T}], \varphi \in \mathrm{C}_{\mathrm{b}, \mathrm{Lip}}\left(\mathbb{R}^{\mathrm{d} \times \mathrm{m}}\right)\right\},
$$

and

$$
\operatorname{Lip}(\Omega):=\bigcup_{n=1}^{\infty} \operatorname{Lip}\left(\Omega_{n}\right),
$$

where $C_{b, \text { Lip }}\left(\mathbb{R}^{d \times m}\right)$ denotes the space of bounded and Lipschitz continuous functions. Let $(B(t))_{t \geqslant 0}$ be real-valued G-Brownian motion on the sublinear expectation space $(\Omega, \mathcal{H}, \overline{\mathbb{E}})$ with $\mathrm{G}(\mathrm{a})=\frac{1}{2}\left(\bar{\sigma}^{2} \mathrm{a}^{+}-\right.$ $\left.\underline{\sigma}^{2} \mathrm{a}^{-}\right)$, where $\bar{\sigma}^{2}=\overline{\mathbb{E}}\left[\mathrm{B}(1)^{2}\right], \underline{\sigma}^{2}=-\overline{\mathbb{E}}\left[-\mathrm{B}(1)^{2}\right], 0 \leqslant \underline{\sigma} \leqslant \bar{\sigma}<\infty$. For $1 \leqslant p<\infty$, let $\mathrm{L}_{\mathrm{G}}^{\mathrm{p}}(\Omega)$ be the completion of $\operatorname{Lip}(\Omega)$ under the norm $\|\cdot\|_{p}=\overline{\mathbb{E}}\left[|\cdot|^{p}\right]^{\frac{1}{p}}$. Similarly, we can define $\mathrm{L}_{\mathrm{G}}^{\mathrm{p}}\left(\Omega_{\mathrm{T}}\right)$ for each $\mathrm{T}>0$. Define the space $M_{G}^{p, 0}([0, T])$ of simple processes by 


$$
\begin{aligned}
M_{G}^{p, 0}([0, T])=\left\{\eta_{t}(\omega)=\sum_{j-1}^{N-1} \xi_{t_{j}}(\omega) I_{\left[t_{j}, t_{j+1}\right.} ; \xi_{t_{j}}(\omega) \in L_{G}^{p}\left(\Omega_{t_{j}}\right),\right. \\
\\
\left.\quad \forall N \geqslant 1,0=t_{0}<t_{1}<\cdots<t_{N}=T, j=0,1, \cdots, N-1\right\} .
\end{aligned}
$$

Let $M_{G}^{p}([0, T])$ be the completion of $M_{G}^{p, 0}([0, T])$ with the norm

$$
\|\eta\|_{M_{G}^{p}([0, T])}=\left(\overline{\mathbb{E}}\left(\int_{0}^{T}\left|\eta_{t}\right|^{p} d t\right)\right)^{\frac{1}{p}}
$$

For the construction of the stochastic integrals with respect to the G-Brownian motion, we can refer to Peng [24]. For each fixed $\tau \in(0, \infty)$, it is referred to as the delay or time lag. Let $\mathscr{C}=\mathrm{C}\left([-\tau, 0] ; \mathbb{R}^{n}\right)$ be the family of all real-valued continuous functions on $[-\tau, 0]$ equipped with the uniform norm $\|\xi\|_{\infty}:=$ $\sup _{-\tau \leqslant \theta \leqslant 0}|\xi(\theta)|$ for $\xi \in \mathscr{C}$. For $X(\cdot) \in \mathrm{C}\left([-\tau, \infty] ; \mathbb{R}^{n}\right)$, define the segment process $X_{t} \in \mathscr{C}$ by

$$
X_{t}(\theta):=X(t+\theta), \quad \theta \in[-\tau, 0], \quad t \geqslant 0 .
$$

Let $\mu(\cdot)$ and $\rho(\cdot)$ denote the finite signed measures on $[-\tau, 0], v(\cdot)$ a measure on $[-\tau, 0], \mathbb{C}$ the set of all complex numbers and $\operatorname{Re}(z)$ the real part of $z \in \mathbb{C}$. We define the G-capacity by $\hat{C}(A)=\sup _{P \in \mathcal{P}} P(A)$, $A \in \mathcal{B}(\Omega)$, where $\mathcal{P}$ is a (weakly compact) collection of probability measures on $(\Omega, \mathcal{B}(\Omega)$ ).

Consider the following deterministic linear retarded equation of neutral type:

$$
\mathrm{d}\left(\mathrm{Y}(\mathrm{t})-\int_{[-\tau, 0]} \mathrm{Y}(\mathrm{t}+\theta) \rho(\mathrm{d} \theta)\right)=\left(\int_{[-\tau, 0]} \mathrm{Y}(\mathrm{t}+\theta) \mu(\mathrm{d} \theta)\right) \mathrm{dt},
$$

with the initial value $Y_{0}=\xi \in \mathscr{C}$. By the variation-of-constants formula (see, e.g., [9, Theorem 1.1, p.256]), (2.1) has a unique solution $\{\mathrm{Y}(\mathrm{t} ; \xi)\}_{\mathrm{t} \geqslant-\tau}$. Set

$$
v:=\sup \left\{\operatorname{Re}(\lambda): \lambda \in \mathbb{C}, \operatorname{det}\left(\lambda\left(I_{n \times n}-\int_{[-\tau, 0]} e^{\lambda \theta} \rho(d \theta)\right)-\int_{[-\tau, 0]} e^{\lambda \theta} \mu(d \theta)\right)=0\right\},
$$

where $\operatorname{det}(A)$ denotes the determinant of an $A \in \mathbb{R}^{n} \otimes \mathbb{R}^{n}$. The fundamental solution of (2.1) is a unique continuous function $G:[0, \infty) \mapsto \mathbb{R}^{n} \otimes \mathbb{R}^{n}$ which satisfying

$$
d\left(G(t)-\int_{[-\tau, 0]} G(t+\theta) \rho(d \theta)\right)=\left(\int_{[-\tau, 0]} G(t+\theta) \mu(d \theta)\right) d t, \quad t>0,
$$

with the initial value $G(0)=I_{n \times n}$ and, for each $\theta \in[-\tau, 0), G(\theta)=0_{n \times n}$. Then according to [1, Lemma $1]$, for any $\alpha>v$, there exists a constant $c_{\alpha}>0$ such that

$$
\|G(t)\| \leqslant c_{\alpha} e^{\alpha t}, \quad t \geqslant-\tau,
$$

where $\|\cdot\|$ denotes the operator norm of the matrix.

Now, we end this subsection by stating the following definitions and a lemma which plays an important role in obtaining our result.

Lemma 2.1 (Peng [24, (4.10), p. 47]). For each $\eta \in M_{G}^{1}(0, T)$,

$$
\overline{\mathbb{E}}\left|\int_{0}^{T} \eta(\mathrm{t}) d\langle\mathrm{~B}, \mathrm{~B}\rangle(\mathrm{t})\right| \leqslant \bar{\sigma}^{2} \overline{\mathbb{E}}\left[\int_{0}^{T}|\eta(\mathrm{t})| d \mathrm{t}\right],
$$

where $\langle\mathrm{B}, \mathrm{B}\rangle(\mathrm{t})$ is the quadratic variation process of $\mathrm{B}(\mathrm{t})$.

Lemma 2.2 (Peng [24, exercise 3.9, p. 45]). For each $\eta \in M_{G}^{p}(0, T)$,

$$
\overline{\mathbb{E}}\left[\int_{0}^{T}|\eta(t)|^{p} d t\right] \leqslant \int_{0}^{T} \overline{\mathbb{E}}|\eta(t)|^{p} d t .
$$


Definition 2.3. The solution $(X(t))$ is said to be $p$-th moment exponentially stable if there is a pair of positive constants $\mathrm{K}$ and $\mathrm{K}$, for any $\mathrm{p}>0$ and $\xi \in \mathscr{C}$ such that

$$
\overline{\mathbb{E}}|X(t ; \xi)|^{p} \leqslant K\|\xi\|_{\infty}^{p} e^{-\kappa p t} .
$$

When $p=2$, it is often called to be exponentially stable in mean square.

Definition 2.4. The solution $(X(t))$ is said to be quasi surely exponentially stable if for any $\xi \in \mathscr{C}$ such that

$$
\limsup _{t \rightarrow \infty} \frac{1}{t} \log |X(t ; \xi)|<0 \quad \text { q.s. . }
$$

\section{Exponential stability of the solutions}

In this work, we first consider the following semi-linear retarded SDE of the form

$$
d\left(X(t)-\int_{[-\tau, 0]} X(t+\theta) \rho(d \theta)\right)=\left(\int_{[-\tau, 0]} X(t+\theta) \mu(d \theta)\right) d t+\sigma\left(X_{t}\right) d B(t),
$$

with the initial value $X_{0}=\xi \in \mathscr{C}$, where $\sigma: \mathscr{C} \mapsto \mathbb{R}$ such that $\sigma(0)=0$ is Borel measurable. Throughout this section, we further assume that the initial value $\xi \in \mathscr{C}$ is independent of $\{B(t)\}_{t} \geqslant 0$, and for any $\phi, \varphi \in \mathscr{C}$ there exists an $\mathrm{L}>0$ such that

$$
|\sigma(\phi)-\sigma(\varphi)|^{2} \leqslant \mathrm{~L}\left(|\phi(0)-\varphi(0)|^{2}+\int_{[-\tau, 0]}|\phi(\theta)-\varphi(\theta)|^{2} v(\mathrm{~d} \theta)\right) .
$$

We remark that the right-hand side of (3.1) do not involve information on current time. We use the variation-of-constants formula to overcome this difficulties. That has been applied successfully in [5]. The following lemma gives a variation-of-constants formula for (3.1).

Lemma 3.1. For each $\xi \in \mathcal{C}$ such that $\int_{[-\tau, 0]}\left|\xi^{\prime}(\theta)\right|^{2} d \theta<\infty$, there is a unique strong solution $\{X(t ; \xi)\}_{\mathfrak{t}>0}$, which can be represented by

$$
\begin{aligned}
X(t ; \xi)= & G(t) \xi(0)-\int_{[-\tau, 0]} G(t+\theta) \xi(0) \rho(d \theta) \\
& +\int_{[-\tau, 0]} \mu(d \theta) \int_{\theta}^{0} G(t+\theta-s) \xi(s) d s \\
& +\int_{[-\tau, 0]} \rho(d \theta) \int_{\theta}^{0} G(t+\theta-s) \xi^{\prime}(s) d s+\int_{0}^{t} G(t-s) \sigma\left(X_{s}\right) d B(s) .
\end{aligned}
$$

Proof. By [17, Theorem 2.2], the solution of (2.1) can be expressed by

$$
\begin{aligned}
Y(t ; \xi)= & G(t) \xi(0)-\int_{[-\tau, 0]} G(t+\theta) \xi(0) \rho(d \theta)+\int_{[-\tau, 0]} \mu(d \theta) \int_{\theta}^{0} G(t+\theta-s) \xi(s) d s \\
& +\int_{[-\tau, 0]} \rho(d \theta) \int_{\theta}^{0} G(t+\theta-s) \xi^{\prime}(s) d s .
\end{aligned}
$$

Moreover, by [1, Theorem 1] it follows that

$$
\begin{aligned}
X(t ; \xi)= & Y(t ; \xi)+\int_{0}^{t} G(t-s) \sigma\left(X_{s}\right) d B(s) \\
= & G(t) \xi(0)-\int_{[-\tau, 0]} G(t+\theta) \xi(0) \rho(d \theta)+\int_{[-\tau, 0]} \mu(d \theta) \int_{\theta}^{0} G(t+\theta-s) \xi(s) d s \\
& +\int_{[-\tau, 0]} \rho(d \theta) \int_{\theta}^{0} G(t+\theta-s) \xi^{\prime}(s) d s+\int_{0}^{t} G(t-s) \sigma\left(X_{s}\right) d B(s) .
\end{aligned}
$$


For convenience, let $\mathrm{W}^{1,2}([-\tau, 0] ; \mathbb{R})$ denote the Sobolev space consisting of functions $\varphi:[-\tau, 0] \longmapsto \mathbb{R}$ such that $\varphi(\cdot)$ and $\varphi^{\prime}(\cdot)$ belong to $\mathrm{L}^{2}([-\tau, 0] ; \mathbb{R})$. For the rest of the work we assume that the initial value of (3.1) belongs to $W^{1,2}([-\tau, 0] ; \mathbb{R})$ and $v<0$. By (2.2), for any $\beta \in(0,-v)$, there exists a constant $C_{\beta}>0$ such that

$$
\|G(t)\| \leqslant C_{\beta} e^{-\beta t}, \quad t \geqslant-\tau .
$$

The following lemma plays an important role in obtaining the exponential stability of the solutions to (3.1).

Lemma 3.2. Let $\mathrm{p} \geqslant 2, \mathrm{f} \in \mathrm{M}_{\mathrm{G}}^{\mathrm{p}}([0, \mathrm{~T}] ; \mathbb{R})$ and $\mathrm{T} \geqslant \mathrm{t}>0$. Then,

$$
\left(\overline{\mathbb{E}}\left|\int_{0}^{t} r(t-s) f(s) d B(s)\right|^{p}\right)^{\frac{2}{p}} \leqslant \frac{1}{2} p(p-1) \bar{\sigma}^{2} \int_{0}^{t}\left(\bar{E}\|r(t-s) f(s)\|^{p}\right)^{\frac{2}{p}} d s,
$$

provided that the integral on the right hand side is finite for each $\mathrm{t}>0$.

Proof. We shall apply the method used in the argument of [4, Lemma 2.3] to prove this lemma. Let

$$
M(u)=\int_{0}^{t} r(t-s) f(s) d B(s), \quad u \in[0, t] .
$$

By the G-Itô formula, for any $u \in[0, t]$, it follows that

$$
\begin{aligned}
\overline{\mathbb{E}}|M(u)|^{p} & \leqslant\left.\frac{1}{2} p(p-1) \overline{\mathbb{E}}\left|\int_{0}^{u}\right| M(s)\right|^{p-2}\|r(t-s) f(s)\|^{2} d\langle B, B\rangle(s) \mid \\
& \leqslant \frac{1}{2} p(p-1) \bar{\sigma}^{2} \overline{\mathbb{E}}\left[\int_{0}^{u}|M(s)|^{p-2}\|r(t-s) f(s)\|^{2} d s\right] \\
& \leqslant \frac{1}{2} p(p-1) \bar{\sigma}^{2} \int_{0}^{u} \overline{\mathbb{E}}|M(s)|^{p-2}\|r(t-s) f(s)\|^{2} d s \\
& \leqslant \frac{1}{2} p(p-1) \bar{\sigma}^{2} \sup _{0 \leqslant s \leqslant u}\left(\overline{\mathbb{E}}|M(s)|^{p}\right)^{\frac{p-2}{p}} \int_{0}^{u}\left(\overline{\mathbb{E}}\|r(t-s) f(s)\|^{p}\right)^{\frac{2}{p}} d s,
\end{aligned}
$$

where in the second step we have used Lemma 2.1, in the third step utilized Lemma 2.2 and in the last step used the Hölder inequality. Then, we have

$$
\sup _{0 \leqslant s \leqslant u} \overline{\mathbb{E}}|M(s)|^{p} \leqslant \frac{1}{2} p(p-1) \bar{\sigma}^{2} \sup _{0 \leqslant s \leqslant u}\left(\overline{\mathbb{E}}|M(s)|^{p}\right)^{\frac{p-2}{p}} \int_{0}^{u}\left(\overline{\mathbb{E}}\|r(t-s) f(s)\|^{p}\right)^{\frac{2}{p}} d s .
$$

This further implies that

$$
\begin{aligned}
\sup _{0 \leqslant s \leqslant u}\left(\overline{\mathbb{E}}|M(s)|^{p}\right)^{\frac{2}{p}} & \leqslant \frac{1}{2} p(p-1) \bar{\sigma}^{2} \int_{0}^{u}\left(\overline{\mathbb{E}}\|r(t-s) f(s)\|^{p}\right)^{\frac{2}{p}} d s \\
& \leqslant \frac{1}{2} p(p-1) \bar{\sigma}^{2} \int_{0}^{t}\left(\overline{\mathbb{E}}\|r(t-s) f(s)\|^{p}\right)^{\frac{2}{p}} d s .
\end{aligned}
$$

Taking $u \uparrow t$ yields that

$$
\left(\overline{\mathbb{E}}\left|\int_{0}^{t} r(t-s) f(s) d B(s)\right|^{p}\right)^{\frac{2}{p}} \leqslant \frac{1}{2} p(p-1) \bar{\sigma}^{2} \int_{0}^{t}\left(\overline{\mathbb{E}}\|r(t-s) f(s)\|^{p}\right)^{\frac{2}{p}} d s
$$

Remark 3.3. For the estimate of the diffusion term, one of the classical methods is to use some martingale inequalities (e.g., [16, Lemma4.5], [24, p.59, Pro6.4], [8, Theorem 2.1]. For example, by means of martingale inequalities Ren et al. [25] discussed the existence and uniqueness of the solutions to SFDEGs and 
established the exponential estimate of the solution. It should be pointed out that martingale inequalities for G-stochastic integrals play an important role in analyzing the diffusion term therein. However, such a method is not applicable to $\int_{0}^{t} r(t-s) f(s) d B(s)$ because it is not a martingale. Lemma 3.2 can deal with this problem.

Next, we shall investigate the $p$-th moment exponential stability and quasi sure exponential stability for the solution $X(t)$ to $(3.1)$.

Theorem 3.4. Let $\mathrm{p} \geqslant 2$. Assume that (3.2) holds with $\mathrm{L}>0$ such that $\mathrm{p}(\mathrm{p}-1) \overline{\mathrm{\sigma}}^{2} \mathrm{LC}_{\beta}^{2}\left(1+v([-\tau, 0]) e^{2 \beta \tau}\right)<4 \beta$, for $\beta \in(0,-v)$, where $C_{\beta}>0$ is introduced in (3.4). Then for any initial value $\xi \in W^{1,2}([-\tau, 0] ; \mathbb{R})$, the solution of (3.1) is $p$-th moment exponentially stable, i.e., there exist constants $K>0$ and $\tilde{\beta}>0$ such that

$$
\overline{\mathbb{E}}|\mathrm{X}(\mathrm{t} ; \xi)|^{\mathrm{p}} \leqslant \mathrm{K}\|\xi\|_{\infty}^{\mathrm{p}} e^{-\mathrm{p} \tilde{\beta} \mathrm{t}}, \quad \mathrm{t} \geqslant 0 .
$$

Proof. For any $p \geqslant 2$, due to $p(p-1) \bar{\sigma}^{2} \lambda C_{\beta}^{2}\left(1+\rho([-\tau, 0]) e^{2 \alpha \tau}\right)<4 \beta$, we can choose $\gamma_{2}>1$ such that

$$
p(p-1) \bar{\sigma}^{2} \gamma_{2}^{\frac{2}{p}} \lambda C_{\beta}^{2}\left(1+\rho([-\tau, 0]) e^{2 \beta \tau}\right)<4 \beta .
$$

In the sequel, we fix $\gamma_{2}>1$ such that (3.6). Then there exists a $\gamma_{1}>1$ such that

$$
(a+b+c)^{q} \leqslant \gamma_{1}\left(a^{q}+b^{q}\right)+\gamma_{2} c^{q}, \quad q>1, a, b, c \in \mathbb{R}^{+} .
$$

By the elementary inequality:

$$
(a+b)^{\theta} \leqslant a^{\theta}+b^{\theta}, \quad 0<\theta<1, \quad a, b \in \mathbb{R}^{+},
$$

it follows from (3.3) and (3.7) that

$$
\begin{aligned}
\overline{\mathbb{E}}|X(t)|^{p} \leqslant & \bar{\gamma}_{1} \overline{\mathbb{E}}|\mathrm{G}(\mathrm{t}) \xi(0)|^{p}+\bar{\gamma}_{1} \overline{\mathbb{E}}\left|\int_{[-\tau, 0]} \mathrm{G}(\mathrm{t}+\theta) \xi(0) \rho(\mathrm{d} \theta)\right|^{p} \\
& +\bar{\gamma}_{1} \overline{\mathbb{E}}\left|\int_{[-\tau, 0]} \mu(\mathrm{d} \theta) \int_{\theta}^{0} \mathrm{G}(\mathrm{t}+\theta-\mathrm{s}) \xi(\mathrm{s}) \mathrm{d} s\right|^{p} \\
& +\bar{\gamma}_{1} \overline{\mathbb{E}}\left|\int_{[-\tau, 0]}^{0} \rho(\mathrm{d} \theta) \int_{\theta}^{0} \mathrm{G}(\mathrm{t}+\theta-\mathrm{s}) \xi^{\prime}(\mathrm{s}) \mathrm{d} s\right|^{p} \\
& +\bar{\gamma}_{2} \overline{\mathbb{E}}\left|\int_{0}^{\mathrm{t}} \mathrm{G}(\mathrm{t}-\mathrm{s}) \sigma\left(\mathrm{X}_{\mathrm{s}}\right) \mathrm{dB}(\mathrm{s})\right|^{p} \\
= & \sum_{i=1}^{5} \mathrm{~J}_{i} .
\end{aligned}
$$

On one hand, by virtue of Lemma 3.2 and the Hölder inequality, together with (3.2) and (3.4), one has

$$
\begin{aligned}
\left(\overline{\mathbb{E}}\left|\int_{0}^{t} G(t-s) \sigma\left(X_{s}\right) d B(s)\right|^{p}\right)^{\frac{2}{p}} & \leqslant \frac{p}{2}(p-1) \int_{0}^{t}\left(\overline{\mathbb{E}}\left|G(t-s) \sigma\left(X_{s}\right)\right|^{p}\right)^{\frac{2}{p}} d s \\
& \leqslant \frac{p}{2}(p-1) C_{\beta}^{2} \int_{0}^{t} e^{-2 \beta(t-s)}\left(\overline{\mathbb{E}}\left|\sigma\left(X_{s}\right)\right|^{p}\right)^{\frac{2}{p}} d s \\
& \leqslant L \frac{p}{2}(p-1) C_{\beta}^{2} \int_{0}^{t} e^{-2 \beta(t-s)}\left[\overline{\mathbb{E}}\left(|X(s)|^{2}+\int_{[-\tau, 0]}|X(s+\theta)|^{2} v(d \theta)\right)^{\frac{p}{2}}\right]^{\frac{2}{p}} d s \\
& \leqslant L \frac{p}{2}(p-1) C_{\beta}^{2} \int_{0}^{t} e^{-2 \beta(t-s)}\left[\left(\overline{\mathbb{E}}|X(s)|^{p}\right)^{\frac{2}{p}}\right.
\end{aligned}
$$




$$
\begin{aligned}
& \left.+\int_{[-\tau, 0]}\left(\overline{\mathbb{E}}|X(s+\theta)|^{p}\right)^{\frac{2}{p}} v(\mathrm{~d} \theta)\right] \mathrm{d} s \\
& \leqslant L \frac{p}{2}(p-1) C_{\beta}^{2} e^{-2 \beta t} \int_{0}^{t} e^{2 \beta s}\left(\overline{\mathbb{E}}|X(s)|^{p}\right)^{\frac{2}{p}} d s+L \frac{p}{2}(p-1) C_{\beta}^{2} e^{-2 \beta t} \\
& \times \int_{0}^{t} \mathrm{e}^{2 \beta s} \mathrm{ds} \int_{[-\tau, 0]}\left(\overline{\mathbb{E}}|\mathrm{X}(\mathrm{s}+\theta)|^{p}\right)^{\frac{2}{p}} v(\mathrm{~d} \theta) \\
& \leqslant L \frac{p}{2}(p-1) C_{\beta}^{2} e^{-2 \beta t} \int_{0}^{t} e^{2 \beta s}\left(\overline{\mathbb{E}}|X(s)|^{p}\right)^{\frac{2}{p}} d s+L \frac{p}{2}(p-1) C_{\beta}^{2} e^{-2 \beta t} \\
& \times v([-\tau, 0]) \mathrm{e}^{2 \beta \tau} \int_{-\tau}^{t} \mathrm{e}^{2 \beta s}\left(\overline{\mathbb{E}}|X(s)|^{p}\right)^{\frac{2}{p}} \mathrm{ds} \\
& \leqslant \mathrm{L} \frac{\mathrm{p}}{2}(\mathrm{p}-1) \mathrm{C}_{\beta}^{2}\left(1+\mathrm{e}^{2 \beta \tau} v([-\tau, 0])\right) \mathrm{e}^{-2 \beta \mathrm{t}} \int_{0}^{\mathrm{t}} \mathrm{e}^{2 \beta s}\left(\overline{\mathbb{E}}|\mathrm{X}(\mathrm{s})|^{\mathrm{p}}\right)^{\frac{2}{\mathrm{p}}} \mathrm{d} s \\
& +L \frac{p}{2}(p-1) C_{\beta}^{2} e^{-2 \beta t} v([-\tau, 0]) e^{2 \beta \tau}(2 \beta)^{-1}\|\xi\|_{\infty}^{2},
\end{aligned}
$$

where in the fourth step we have used Minkovskii's inequality.

On the other hand, by the Hölder inequality and (3.4), it is readily seen that

$$
\begin{aligned}
\overline{\mathbb{E}}\left|\int_{[-\tau, 0]} \mu(\mathrm{d} \theta) \int_{\theta}^{0} \mathrm{G}(\mathrm{t}+\theta-\mathrm{s}) \xi(\mathrm{s}) \mathrm{d} s\right|^{2} & \leqslant \overline{\mathbb{E}}\left|\mu([-\tau, 0]) \int_{[-\tau, 0]} \mu(\mathrm{d} \theta)\left(\int_{\theta}^{0} \mathrm{G}(\mathrm{t}+\theta-\mathrm{s}) \xi(\mathrm{s}) \mathrm{d} s\right)^{2}\right| \\
& \leqslant \overline{\mathbb{E}}\left|\mu([-\tau, 0]) \int_{[-\tau, 0]}-\theta \mu(\mathrm{d} \theta) \int_{\theta}^{0}\|\mathrm{G}(\mathrm{t}+\theta-\mathrm{s}) \xi(\mathrm{s})\|^{2} \mathrm{~d} s\right| \\
& \leqslant \overline{\mathbb{E}}\left|\mu([-\tau, 0]) \mathrm{C}_{\beta}^{2}\|\xi\|_{\infty}^{2} \int_{[-\tau, 0]}-\theta\left(\int_{\theta}^{0} \mathrm{e}^{-2 \beta(\mathrm{t}+\theta-s)} \mathrm{d} s\right) \mu(\mathrm{d} \theta)\right| \\
& \leqslant(\mu([-\tau, 0]))^{2} \tau \mathrm{C}_{\beta}^{2}\|\xi\|_{\infty}^{2} \mathrm{e}^{-2 \beta \mathrm{t}} \int_{0}^{\tau} \mathrm{e}^{2 \beta s} \mathrm{ds},
\end{aligned}
$$

and

$$
\begin{aligned}
\overline{\mathbb{E}}\left|\int_{[-\tau, 0]} \rho(\mathrm{d} \theta) \int_{\theta}^{0} \mathrm{G}(\mathrm{t}+\theta-\mathrm{s}) \xi^{\prime}(\mathrm{s}) \mathrm{d} s\right|^{2} & \leqslant \rho([-\tau, 0]) \overline{\mathbb{E}} \int_{[-\tau, 0]}\left(\int_{\theta}^{0} \mathrm{G}(\mathrm{t}+\theta-\mathrm{s}) \xi^{\prime}(\mathrm{s}) \mathrm{d} s\right)^{2} \rho(\mathrm{d} \theta) \\
& \leqslant \rho([-\tau, 0]) \overline{\mathbb{E}} \int_{[-\tau, 0]}-\theta \rho(\mathrm{d} \theta) \int_{\theta}^{0}|\mathrm{G}(\mathrm{t}+\theta-s)|^{2}\left|\xi^{\prime}(\mathrm{s})\right|^{2} \mathrm{~d} s \\
& \leqslant \rho([-\tau, 0]) \mathrm{C}_{\beta}^{2} \mathrm{e}^{-2 \beta \mathrm{t}}\left\|\xi^{\prime}\right\|_{\infty}^{2} \int_{[-\tau, 0]}-\theta \rho(\mathrm{d} \theta) \int_{\theta}^{0} \mathrm{e}^{-2 \beta(\theta-s)} \mathrm{d} s \\
& \leqslant(\rho([-\tau, 0]))^{2} \mathrm{C}_{\beta}^{2} \tau \mathrm{e}^{-2 \beta \mathrm{t}}\left\|\xi^{\prime}\right\|_{\infty}^{2} \int_{0}^{\tau} \mathrm{e}^{2 \beta s} \mathrm{ds} .
\end{aligned}
$$

By a slight variation on (3.8) and taking the previous estimates (3.9), (3.10) and (3.11) into consideration, it gives that

$$
\begin{aligned}
\left(\overline{\mathbb{E}}|X(t)|^{p}\right)^{\frac{2}{p}} \leqslant & \bar{\gamma}_{1}^{\frac{2}{p}} C_{\beta}^{2} \mathrm{e}^{-2 \beta \mathrm{t}}\|\xi\|_{\infty}^{2}+\bar{\gamma}_{1}^{\frac{2}{p}}(\rho([-\tau, 0]))^{2} \mathrm{e}^{2 \beta \tau} C_{\beta}^{2} \mathrm{e}^{-2 \beta \mathrm{t}}\|\xi\|_{\infty}^{2} \\
& +\bar{\gamma}_{1}^{\frac{2}{\mathrm{p}}}(\mu([-\tau, 0]))^{2} \tau \mathrm{C}_{\beta}^{2}\|\xi\|_{\infty}^{2} \mathrm{e}^{-2 \beta \mathrm{t}} \int_{0}^{\tau} \mathrm{e}^{2 \beta s} \mathrm{ds} \\
& +\bar{\gamma}_{1}^{\frac{2}{p}}(\rho([-\tau, 0]))^{2} C_{\beta}^{2} \tau \mathrm{e}^{-2 \beta \mathrm{t}}\left\|\xi^{\prime}\right\|_{\infty}^{2} \int_{0}^{\tau} \mathrm{e}^{2 \beta s} \mathrm{ds} \\
& +\bar{\gamma}_{2}^{\frac{2}{\mathrm{p}}} \mathrm{L} \frac{\mathrm{p}}{2}(\mathrm{p}-1) \mathrm{C}_{\beta}^{2} \mathrm{e}^{-2 \beta \mathrm{t}} v([-\tau, 0]) \mathrm{e}^{2 \beta \tau}(2 \beta)^{-1}\|\xi\|_{\infty}^{2} \\
& +\bar{\gamma}_{2}^{\frac{2}{\mathrm{p}}} \mathrm{L} \frac{\mathrm{p}}{2}(\mathrm{p}-1) \mathrm{C}_{\beta}^{2}\left(1+\mathrm{e}^{2 \beta \tau} v([-\tau, 0])\right) \mathrm{e}^{-2 \beta \mathrm{t}} \int_{0}^{\mathrm{t}} \mathrm{e}^{2 \beta \mathrm{s}}\left(\overline{\mathbb{E}}|\mathrm{X}(\mathrm{s})|^{p}\right)^{\frac{2}{\mathrm{p}}} \mathrm{ds},
\end{aligned}
$$


which implies that

$$
\begin{gathered}
\mathrm{e}^{2 \beta \mathrm{t}}\left(\overline{\mathbb{E}}|X(\mathrm{t})|^{\mathrm{p}}\right)^{\frac{2}{\mathrm{p}}} \leqslant \mathrm{K}_{1}+\bar{\gamma}_{2}^{\frac{2}{\mathrm{p}}} \mathrm{L} \frac{\mathrm{p}}{2}(\mathrm{p}-1) \mathrm{C}_{\beta}^{2}\left(1+\mathrm{e}^{2 \beta \tau} v([-\tau, 0])\right) \int_{0}^{\mathrm{t}} \mathrm{e}^{2 \beta s}\left(\overline{\mathbb{E}}|X(s)|^{\mathrm{p}}\right)^{\frac{2}{\mathrm{p}}} \mathrm{d} s, \\
\mathrm{~K}_{1}=\bar{\gamma}_{1}^{\frac{2}{\mathrm{p}}} \mathrm{C}_{\beta}^{2}\|\xi\|_{\infty}^{2}+\bar{\gamma}_{1}^{\frac{2}{\mathrm{p}}}(\rho([-\tau, 0]))^{2} \mathrm{e}^{2 \beta \tau} \mathrm{C}_{\beta}^{2}\|\xi\|_{\infty}^{2}+\bar{\gamma}_{1}(\mu([-\tau, 0]))^{2} \tau \mathrm{C}_{\beta}^{2}\|\xi\|_{\infty}^{2} \int_{0}^{\tau} \mathrm{e}^{2 \beta s} \mathrm{ds} \\
+\bar{\gamma}_{1}^{\frac{2}{\mathrm{p}}}(\rho([-\tau, 0]))^{2} \mathrm{C}_{\beta}^{2} \tau\left\|\xi^{\prime}\right\|_{\infty}^{2} \int_{0}^{\tau} \mathrm{e}^{2 \beta s} \mathrm{~d} s+\bar{\gamma}_{2}^{\frac{1}{\mathrm{p}}} \mathrm{L} \frac{\mathrm{p}}{2}(\mathrm{p}-1) \mathrm{C}_{\beta}^{2} v([-\tau, 0]) \mathrm{e}^{2 \beta \tau}(2 \beta)^{-1}\|\xi\|_{\infty}^{2} .
\end{gathered}
$$

So, the Gronwall inequality leads to

$$
\left(\overline{\mathbb{E}}|X(t)|^{p}\right)^{\frac{2}{p}} \leqslant K_{1} e^{-2 \tilde{\beta} t}, \quad t \geqslant 0,
$$

where $2 \tilde{\beta}:=2 \beta-\bar{\gamma}_{2}^{\frac{2}{p}} L \frac{p}{2}(p-1) C_{\beta}^{2}\left(1+e^{2 \beta \tau} v([-\tau, 0])\right)$. This further gives for $p \geqslant 2$,

$$
\overline{\mathbb{E}}|X(t)|^{p} \leqslant K\|\xi\|_{\infty}^{p} e^{-p \tilde{\beta} t}, \quad t \geqslant 0 .
$$

Note that (3.6) implies that $\tilde{\beta}>0$, therefore the result (3.5) is established in the case of $p \geqslant 2$.

Noting

$$
\left(\overline{\mathbb{E}}|X(t)|^{p}\right)^{\frac{1}{p}} \leqslant\left(\overline{\mathbb{E}}|X(t)|^{\bar{p}}\right)^{\frac{1}{\bar{p}}}, \quad \text { for } 0<p<2, \quad \bar{p} \geqslant 2,
$$

together with (3.5), we see that the $\bar{p}$-th moment exponential stability implies the $p$-th moment exponential stability. Taking $\bar{p}=2$ yields that the estimate to $\overline{\mathbb{E}}|X(t)|^{\mathrm{P}}(0<p<2)$ can be done by exponential stability in mean square. Therefore, we have the following corollary.

Corollary 3.5. Let $0<p<2$. Assume that (3.2) holds with $\mathrm{L}>0$ such that $\mathrm{L}^{2} \mathrm{C}_{\beta}^{2}\left(1+v([-\tau, 0]) e^{2 \beta \tau}\right)<2 \beta$, for $\beta \in(0,-v)$, where $C_{\beta}>0$ is introduced in (3.4). Then for any initial value $\xi \in W^{1,2}([-\tau, 0] ; \mathbb{R})$, the solution of (3.1) is $p$-th moment exponentially stable, i.e., there exist constants $\mathrm{K}>0$ and $\beta>0$ such that

$$
\overline{\mathbb{E}}|\mathrm{X}(\mathrm{t} ; \xi)|^{\mathrm{p}} \leqslant \mathrm{K}\|\xi\|_{\infty}^{\mathrm{p}} e^{-\mathrm{p} \beta \mathrm{t}}, \mathrm{t} \geqslant 0 .
$$

Carrying out similar arguments as Theorem 3.4 and Corollary 3.5, we can show that the solution $X(t)$ of (3.1) has the property as follows:

Theorem 3.6. Let the conditions of theorem 3.4 hold. Then for any different initial values $\xi, \eta \in W^{1,2}([-\tau, 0] ; \mathbb{R})$, there exists a pair of positive constants $\mathrm{K}$ and $\tilde{\beta}$ such that

$$
\overline{\mathbb{E}}|X(t ; \xi)-X(t ; \eta)|^{p} \leqslant K\|\xi-\eta\|_{\infty}^{p} e^{-p \tilde{\beta} t}, \quad t \geqslant 0, \quad p \geqslant 2,
$$

where $\tilde{\beta}$ is given in Theorem 3.4 .

Corollary 3.7. Let the conditions corollary 3.5 hold. Then for any different initial values $\xi, \eta \in W^{1,2}([-\tau, 0] ; \mathbb{R})$, there exists a pair of positive constants $\mathrm{K}$ and $\beta$ such that

$$
\overline{\mathbb{E}}|X(\mathrm{t} ; \xi)-X(\mathrm{t} ; \eta)|^{\mathrm{p}} \leqslant \mathrm{K}\|\xi-\eta\|_{\infty}^{\mathrm{p}} e^{-\mathrm{p} \beta \mathrm{t}}, \quad \mathrm{t} \geqslant 0,0<\mathrm{p}<2 .
$$

In a stable system, by virtue of the results of Theorem 3.6 and Corollary 3.7, trajectories of solutions corresponding to different initial values become closer in the sense of $p$-th moment after a long time.

If some conditions are required, moment exponential stability can imply quasi sure exponential stability. The following result demonstrates this point.

Theorem 3.8. Let the conditions of theorem 3.4 hold. Then for any initial values $\xi \in W^{1,2}([-\tau, 0] ; \mathbb{R})$, there exists a constant $\gamma>0$ such that

$$
\limsup _{t \rightarrow \infty} \frac{1}{t} \ln |X(t)| \leqslant-\gamma, \text { q.s., }
$$

where $\gamma=\min \left\{2 \tilde{\beta}, \tau^{-1} \ln \frac{1}{(\rho([-\tau, 0]))^{2}}\right\}, \tilde{\beta}$ is given in Theorem 3.4. 
Proof. For any integer $n \geqslant 1$, using the Doob martingale inequality and Hölder's inequality, together with (3.1), (3.2) and (3.5), we have

$$
\begin{aligned}
& \overline{\mathbb{E}}\left(\sup _{0 \leqslant \bar{\theta} \leqslant \tau}\left|X(n \tau+\bar{\theta})-\int_{[-\tau, 0]} X(n \tau+\theta+\bar{\theta}) \rho(d \theta)\right|^{2}\right) \\
& \leqslant 3 \overline{\mathbb{E}}\left|X(n \tau)-\int_{[-\tau, 0]} X(n \tau+\theta) \rho(d \theta)\right|^{2}+3 \overline{\mathbb{E}}\left|\int_{n \tau}^{(n+1) \tau}\left(\int_{[-\tau, 0]} X(s+\theta) \mu(d \theta)\right) d s\right|^{2} \\
& +3 \overline{\mathbb{E}}\left(\sup _{0 \leqslant \bar{\theta} \leqslant \tau}\left|\int_{n \tau}^{n \tau+\bar{\theta}} \sigma\left(X_{s}\right) \mathrm{d} W(s)\right|^{2}\right) \\
& \leqslant 6 \overline{\mathbb{E}}|X(n \tau)|^{2}+6 \overline{\mathbb{E}}\left|\int_{[-\tau, 0]} X(n \tau+\theta) \rho(d \theta)\right|^{2}+3 \tau \overline{\mathbb{E}} \int_{n \tau}^{(n+1) \tau}\left|\int_{[-\tau, 0]} X(s+\theta) \mu(d \theta)\right|^{2} d s \\
& +12 \int_{n \tau}^{(n+1) \tau} \overline{\mathbb{E}}\|\sigma(X(s+\theta))\|^{2} \mathrm{ds} \\
& \leqslant 6 \overline{\mathbb{E}}|X(n \tau)|^{2}+6 \rho([-\tau, 0]) \int_{[-\tau, 0]} \overline{\mathbb{E}}|X(n \tau+\theta)|^{2} \rho(d \theta)+3 \tau \mu([-\tau, 0]) \\
& \times \overline{\mathbb{E}} \int_{n \tau}^{(n+1) \tau} \int_{[-\tau, 0]}|X(s+\theta)|^{2} \mu(d \theta) d s \\
& +12 \mathrm{~L} \overline{\mathbb{E}} \int_{n \tau}^{(n+1) \tau}\left(|X(s)|^{2}+\int_{[-\tau, 0]}|X(s+\theta)|^{2} v(d \theta)\right) d s \\
& \leqslant 6 \overline{\mathbb{E}}|X(n \tau)|^{2}+6(\rho([-\tau, 0]))^{2} \sup _{-\tau \leqslant \theta \leqslant 0} \overline{\mathbb{E}}|X(n \tau+\theta)|^{2}+3 \tau \mu([-\tau, 0]) \\
& \times \int_{[-\tau, 0]} \mu(\mathrm{d} \theta) \int_{n \tau}^{(n+1) \tau} \overline{\mathbb{E}}|X(s+\theta)|^{2} \mathrm{~d} s \\
& +12 L \int_{n \tau}^{(n+1) \tau} \overline{\mathbb{E}}|X(s)|^{2} d s+12 L \int_{n \tau}^{(n+1) \tau}\left(\int_{[-\tau, 0]} \overline{\mathbb{E}}|X(s+\theta)|^{2} v(d \theta)\right) d s \\
& \leqslant 6 \overline{\mathbb{E}}|X(n \tau)|^{2}+6(\rho([-\tau, 0]))^{2} \sup _{-\tau \leqslant \theta \leqslant 0} \overline{\mathbb{E}}|X(n \tau+\theta)|^{2}+3 \tau(\mu([-\tau, 0]))^{2} \int_{n \tau-\tau}^{(n+1) \tau} \overline{\mathbb{E}}|X(s)|^{2} d s \\
& +12 L \int_{n \tau}^{(n+1) \tau} \overline{\mathbb{E}}|X(s)|^{2} \mathrm{~d} s+12 \mathrm{~L} v([-\tau, 0]) \int_{n \tau-\tau}^{(n+1) \tau} \overline{\mathbb{E}}|X(s)|^{2} \mathrm{~d} s \\
& \leqslant 6 K\|\xi\|_{\infty}^{2} \mathrm{e}^{-\bar{\gamma} n \tau}+6(\rho([-\tau, 0]))^{2} \mathrm{~K}\|\xi\|_{\infty}^{2} \mathrm{e}^{-\bar{\gamma}(n \tau-\tau)}+3 \tau(\mu([-\tau, 0]))^{2} \mathrm{~K}\|\xi\|_{\infty}^{2} \int_{n \tau-\tau}^{(n+1) \tau} \mathrm{e}^{-\bar{\gamma} s} \mathrm{~d} s \\
& +12 \mathrm{LK}\|\xi\|_{\infty}^{2} \int_{n \tau}^{(n+1) \tau} \mathrm{e}^{-\bar{\gamma} s} \mathrm{ds}+12 \operatorname{L} v([-\tau, 0]) K\|\xi\|_{\infty}^{2} \int_{n \tau-\tau}^{(n+1) \tau} \mathrm{e}^{-\bar{\gamma} s} \mathrm{ds} \\
& \leqslant 6 \mathrm{~K}\|\xi\|_{\infty}^{2} \mathrm{e}^{-\bar{\gamma} n \tau}+6(\rho([-\tau, 0]))^{2} \mathrm{~K}\|\xi\|_{\infty}^{2} \mathrm{e}^{\bar{\gamma} \tau} \mathrm{e}^{-\bar{\gamma} n \tau}+3 \tau(\mu([-\tau, 0]))^{2} \mathrm{~K}\|\xi\|_{\infty}^{2} \bar{\gamma}^{-1} \mathrm{e}^{\bar{\gamma} \tau} \mathrm{e}^{-\bar{\gamma} n \tau} \\
& +12 \operatorname{LK}\|\xi\|_{\infty}^{2} \bar{\gamma}^{-1} \mathrm{e}^{-\bar{\gamma} n \tau}+12 \operatorname{Lv}([-\tau, 0]) \mathrm{K}\|\xi\|_{\infty}^{2} \bar{\gamma}^{-1} \mathrm{e}^{\bar{\gamma} \tau} \mathrm{e}^{-\bar{\gamma} n \tau} \\
& =\mathrm{Ce}^{-\bar{\gamma} n \tau} \text {, }
\end{aligned}
$$

where

$$
\mathrm{C}=\mathrm{K}\|\xi\|_{\infty}^{2}\left[6+6(\rho([-\tau, 0]))^{2} \mathrm{e}^{\bar{\gamma} \tau}+3 \tau(\mu([-\tau, 0]))^{2} \bar{\gamma}^{-1} \mathrm{e}^{\bar{\gamma} \tau}+12 \mathrm{~L} \bar{\gamma}^{-1}+12 \mathrm{~L} v([-\tau, 0]) \bar{\gamma}^{-1} \mathrm{e}^{\bar{\gamma} \tau}\right] .
$$

For any $\varepsilon \in(0, \bar{\gamma})$, using the Chebyshev inequality, we have

$$
\hat{C}\left(\omega: \sup _{0 \leqslant \bar{\theta} \leqslant \tau}\left|X(n \tau+\bar{\theta})-\int_{[-\tau, 0]} X(n \tau+\theta+\bar{\theta}) \rho(d \theta)\right|^{2}>\mathrm{e}^{-(\bar{\gamma}-\varepsilon) n \tau}\right) \leqslant C \mathrm{e}^{-\varepsilon n \tau} .
$$


The Borel-Cantelli lemma for capacity [6] yields that for almost all $\omega \in \Omega$, there exists an integer $n_{0}(\omega)$ such that

$$
\sup _{0 \leqslant \bar{\theta} \leqslant \tau}\left|X(n \tau+\bar{\theta})-\int_{[-\tau, 0]} X(n \tau+\theta+\bar{\theta}) \rho(d \theta)\right|^{2} \leqslant e^{-(\bar{\gamma}-\varepsilon) n \tau}, \quad n \geqslant n_{0} .
$$

Consequently, for almost all $\omega \in \Omega$, if $t \geqslant n_{0} \tau$,

$$
\left|X(t)-\int_{[-\tau, 0]} X(t+\theta) \rho(d \theta)\right|^{2} \leqslant e^{-(\bar{\gamma}-\varepsilon)(t-\tau)} .
$$

Moreover, for $t \in\left[0, n_{0} \tau\right],\left|X(t)-\int_{[-\tau, 0]} X(t+\theta) \rho(d \theta)\right|^{2}$ is finite. So, for almost all $\omega \in \Omega$, there exists a finite constant $H=H(\omega)$, if $t \geqslant 0$,

$$
\left|X(t)-\int_{[-\tau, 0]} X(t+\theta) \rho(d \theta)\right|^{2} \leqslant \mathrm{He}^{-(\bar{\gamma}-\varepsilon) t} .
$$

On the other hand, set $\mathrm{e}^{\bar{\gamma} \tau}(\rho([-\tau, 0]))^{2}<\varepsilon<1$. For $\mathrm{t} \geqslant 0$, note that

$$
\begin{aligned}
\left|X(t)-\int_{[-\tau, 0]} X(t+\theta) \rho(d \theta)\right|^{2} & \geqslant|X(t)|^{2}-2|X(t)|\left|\int_{[-\tau, 0]} X(t+\theta) \rho(d \theta)\right|+\left|\int_{[-\tau, 0]} X(t+\theta) \rho(d \theta)\right|^{2} \\
& \geqslant(1-\varepsilon)|X(t)|^{2}+\left(\varepsilon^{-1}-1\right)\left|\int_{[-\tau, 0]} X(t+\theta) \rho(d \theta)\right|^{2} .
\end{aligned}
$$

Hence, we see that

$$
|X(t)|^{2} \leqslant \frac{1}{1-\varepsilon}\left|X(t)-\int_{[-\tau, 0]} X(t+\theta) \rho(d \theta)\right|^{2}+\frac{1}{\varepsilon}\left|\int_{[-\tau, 0]} X(t+\theta) \rho(d \theta)\right|^{2} .
$$

Also, for each $\mathrm{T}>0$,

$$
\begin{aligned}
\sup _{0 \leqslant t \leqslant T}\left[e^{(\bar{\gamma}-\varepsilon) t}|X(t)|^{2}\right] & \leqslant \frac{H}{1-\varepsilon}+\frac{1}{\varepsilon} \sup _{0 \leqslant t \leqslant T}\left[e^{(\bar{\gamma}-\varepsilon) t}\left|\int_{[-\tau, 0]} X(t+\theta) \rho(d \theta)\right|^{2}\right] \\
& \leqslant \frac{H}{1-\varepsilon}+\frac{e^{(\bar{\gamma}-\varepsilon) \tau}(\rho([-\tau, 0]))^{2}}{\varepsilon} \sup _{-\tau \leqslant t \leqslant T}\left[e^{(\bar{\gamma}-\varepsilon) t}|X(t)|^{2}\right] .
\end{aligned}
$$

Through a straightforward mathematical computation, we get

$$
\left(1-\frac{\mathrm{e}^{(\bar{\gamma}-\varepsilon) \tau}(\rho([-\tau, 0]))^{2}}{\varepsilon}\right) \sup _{0 \leqslant \mathrm{t} \leqslant \mathrm{T}}\left[\mathrm{e}^{(\bar{\gamma}-\varepsilon) \mathrm{t}}|X(\mathrm{t})|^{2}\right] \leqslant \frac{\mathrm{H}}{1-\varepsilon}+\frac{\mathrm{e}^{(\bar{\gamma}-\varepsilon) \tau}(\rho([-\tau, 0]))^{2}}{\varepsilon}\|\xi\|_{\infty}^{2},
$$

which implies that

$$
\limsup _{t \rightarrow \infty} \frac{1}{t} \ln |X(t)| \leqslant-\frac{\bar{\gamma}-\varepsilon}{2} \text { a.s. . }
$$

Let $\varepsilon \rightarrow 0$. The required result is obtained.

\section{An illustrative example}

In this section, we consider an example to verify the theory established in the previous section.

Example 4.1. Consider a semi-linear retarded SDEs

$$
d\left(X(t)+\frac{1}{3} X(t-1)\right)=-X(t-1) d t+\sigma(X(t-1)) d B(t), \quad X_{0}=\xi \in \mathscr{C} .
$$

In view of the corresponding characteristic equation $\lambda+\left(1+\frac{\lambda}{3}\right) e^{-\lambda}=0$, we deduce that the unique root is $\lambda \approx-2.3134$. So, we have $v=-2.3134$. Therefore, taking $p=2$ and by Theorem 3.4, when the Lipschitz constant $L$ of $\sigma$ such that $\operatorname{Lo}^{2} C_{\beta}^{2}\left(1+v([-\tau, 0]) \mathrm{e}^{2 \beta \tau}\right)<2 \beta$, for $\beta \in(0,2.3134)$, the solution $X(t)$ of $(4.1)$ is quasi sure exponentially stable and exponentially stable in mean square. 


\section{Acknowledgment}

We are grateful to the referees for their many valuable comments and suggestions which improved this article. This work was supported by the National Natural Science Foundation of China (No. 11371374 and 11571372), the Natural Science Foundation of Hunan University of Technology (2012HZX08), and the Special Foundation of National independent innovation demonstration area construction of Zhuzhou (applied basic research).

\section{References}

[1] J. A. D. Appleby, H.-Z. Appleby-Wu, X.-R. Mao, On the almost sure running maxima of solutions of affine neutral stochastic functional differential equations, ArXiv, 2013 (2013), 28 pages. 2, 3

[2] J.-H. Bao, Z.-T. Hou, C.-G. Yuan, Stability in distribution of neutral stochastic differential delay equations with Markovian switching, Statist. Probab. Lett., 79 (2009), 1663-1673. 1

[3] J.-H. Bao, G. Yin, C.-G. Yuan, Ergodicity for functional stochastic differential equations and applications, Nonlinear Anal., 98 (2014), 66-82. 1

[4] J.-H. Bao, G. Yin, C.-G. Yuan, Asymptotic analysis for functional stochastic differential equations, SpringerBriefs in Mathematics, Springer, Cham, (2016). 3

[5] J.-H. Bao, G. Yin, C.-G. Yuan, Stationary distributions for retarded stochastic differential equations without dissipativity, Stochastics, 89 (2016), 530-549. 1, 3

[6] Z.-J. Chen, Strong laws of large numbers for capacities, ArXiv, 2010 (2010), 17 pages. 3

[7] W.-Y. Fei, C. Fei, On exponential stability for stochastic differential equations disturbed by G-Brownian motion, ArXiv, 2013 (2013), 19 pages. 1

[8] F.-Q. Gao, Pathwise properties and homeomorphic flows for stochastic differential equations driven by G-Brownian motion, Stochastic Process. Appl., 119 (2009), 3356-3382. 3.3

[9] J. K. Hale, S. M. Verduyn Lunel, Introduction to functional-differential equations, Applied Mathematical Sciences, Springer-Verlag, New York, (1993). 1, 2

[10] Z.-T. Hou, J.-H. Bao, C.-G. Yuan, Exponential stability of energy solutions to stochastic partial differential equations with variable delays and jumps, J. Math. Anal. Appl., 366 (2010), 44-54. 1

[11] L.-Y. Hu, Y. Ren, T.-B. Xu, p-moment stability of solutions to stochastic differential equations driven by G-Brownian motion, Appl. Math. Comput., 230 (2014), 231-237. 1

[12] S. Janković, J. Randjelovic, M. Jovanović, Razumikhin-type exponential stability criteria of neutral stochastic functional differential equations, J. Math. Anal. Appl., 355 (2009), 811-820. 1

[13] N. N. Krasovskir, Stability of motion, Applications of Lyapunov's second method to differential systems and equations with delay, Translated by J. L. Brenner, Stanford University Press, Stanford, Calif., (1963). 1

[14] H. J. Kushner, On the stability of processes defined by stochastic difference-differential equations, J. Differential Equations, 4 (1968), 424-443. 1

[15] T. Li, J. Wei, Y.-Q. Suo, Stability in distribution of neutral stochastic functional differential equations, Statist. Probab. Lett., 107 (2015), 27-36. 1

[16] Q. Lin, Local time and Tanaka formula for the G-Brownian motion, J. Math. Anal. Appl., 398 (2013), 315-334. 3.3

[17] K. Liu, The fundamental solution and its role in the optimal control of infinite dimensional neutral systems, Appl. Math. Optim., 60 (2009), 1-38. 1, 3

[18] Q. Luo, X.-R. Mao, Y. Shen, New criteria on exponential stability of neutral stochastic differential delay equations, Systems Control Lett., 55 (2006), 826-834. 1

[19] X.-R. Mao, Exponential stability in mean square of neutral stochastic differential-functional equations, Systems Control Lett., 26 (1995), 245-251. 1

[20] X.-R. Mao, Razumikhin-type theorems on exponential stability of neutral stochastic functional-differential equations, SIAM J. Math. Anal., 28 (1997), 389-401. 1

[21] X.-R. Mao, Asymptotic properties of neutral stochastic differential delay equations, Stochastics Stochastics Rep., 68 (2000), 273-295. 1

[22] S.-G. Peng, G-expectation, G-Brownian motion and related stochastic calculus of Itô type, Stochastic analysis and applications, Abel Symp., Springer, Berlin, 2 (2007), 541-567. 1

[23] S.-G. Peng, Multi-dimensional G-Brownian motion and related stochastic calculus under G-expectation, Stochastic Process. Appl., 118 (2008), 2223-2253. 1

[24] S.-G. Peng, Nonlinear expectations and stochastic calculus under uncertainty, ArXiv, 2010 (2010), 149 pages. 1, 2, 2.1, $2.2,3.3$

[25] Y. Ren, Q. Bi, R. Sakthivel, Stochastic functional differential equations with infinite delay driven by G-Brownian motion, Math. Methods Appl. Sci., 36 (2013), 1746-1759. 1, 3.3

[26] Y. Ren, X.-J. Jia, L.-Y. Hu, Exponential stability of solutions to impulsive stochastic differential equations driven by GBrownian motion, Discrete Contin. Dyn. Syst. Ser. B, 20 (2015), 2157-2169. 1 
[27] D.-F. Zhang, Z.-J. Chen, Exponential stability for stochastic differential equation driven by G-Brownian motion, Appl. Math. Lett., 25 (2012), 1906-1910. 1

[28] X.-F. Zong, F. Wu, Exponential stability of the exact and numerical solutions for neutral stochastic delay differential equations, Appl. Math. Model., 40 (2016), 19-30. 1 